\title{
Magnetic alginate microspheres detected by MRI fabricated using microfluidic technique and release behavior of encapsulated dual drugs
}

This article was published in the following Dove Press journal:

International Journal of Nanomedicine

8 June 2017

Number of times this article has been viewed

\author{
Qin Wang' \\ Shanshan Liu' \\ Fan Yang ${ }^{2}$ \\ Lu Gan ${ }^{3}$ \\ Xiangliang Yang ${ }^{3}$ \\ Yajiang Yang' \\ 'National Engineering Research \\ Center for Nanomedicine, School of \\ Chemistry and Chemical Engineering, \\ ${ }^{2}$ Department of Radiology, Union \\ Hospital, Tongji Medical College, \\ ${ }^{3}$ National Engineering Research \\ Center for Nanomedicine, College of \\ Life Science and Technology, Huazhong \\ University of Science and Technology, \\ Wuhan, China
}

\begin{abstract}
Alginate microspheres loaded with superparamagnetic iron oxide nanoparticles (SPIO NPs) have been fabricated by a T-junction microfluidic device combined with an external ionic crosslinking. The obtained microspheres possess excellent visuality under magnetic resonance due to the presence of only $0.6 \mathrm{mg} / \mathrm{mL}$ SPIO NPs. The microspheres also show uniform size with narrow distribution and regular spherical shape characterized by optic microscope and environmental scanning electron microscope. Furthermore, dual drugs (5-fluorouracil and doxorubicin hydrochloride) have been loaded within the microspheres. The release behavior of dual drugs from the microspheres show typical sustained release profiles. As a novel embolic agent, such microspheres in blood vessels can be tracked by magnetic resonance scanner. Thus, the integration of embolotherapy, chemotherapy, and postoperative diagnosis can be realized. Keywords: MRI visuality, chemoembolization, microfluidics, dual drugs, alginate microspheres
\end{abstract}

\section{Introduction}

Transcatheter arterial embolization (TAE) is an effective method for the tumor therapy. The principle of TAE is based on the embolization of the tumor feeding arteries using embolic agents. Thus the supply of nutrition to the tumor is blocked, resulting in tumor necrosis. ${ }^{1,2}$ In the past decade, an improved TAE was developed known as transcatheter arterial chemoembolization (TACE), in which anticancer drugs were loaded within the embolic agents. Both embolization and chemotherapy are combined in TACE. In comparison with conventional chemotherapy, TACE not only enhances drug targeting but also shows better efficiency and less side effects. Therefore, TACE has been accepted as the preferred therapy of the unresectable liver cancer ${ }^{3-5}$ However, a major problem with both TAE and TACE is how to determine the location of the embolic agents in blood vessels and evaluate the effect of embolization. Conventional assessment method is based on the blood flow via angiography, but the reliability is questioned. For example, in the treatment of uterine fibroids, angiography indicated that the blood vessels in the tumor were completely embolized. In fact, $20 \%$ of the blood vessels were not embolized yet, leading to the regeneration of tumor cells. ${ }^{6}$ However, if excessive embolic agents were injected, uterine injury may occur. ${ }^{7}$ Consequently, improvement of visuality of the embolic agents still remains a challenge in the field of embolization therapy.

Lipiodol $^{\circledR}$ (ethiodized poppyseed oil), alone or mixed with other embolic agents, is currently used as the embolic agent due to its X-ray radiopacity. ${ }^{8}$ However, its natural 
liquid state means it easily diffuses or dissociates from the blood vessel. We previously reported barium alginate microspheres and poly $(N$-isopropylacrylamide $)$ microspheres encapsulated with in situ-synthesized $\mathrm{BaSO}_{4}$ nanoparticles. ${ }^{9-11}$ These microspheres show excellent visuality under the $\mathrm{X}$-ray. Yet, the disadvantage of X-ray imaging technology is the potential radiation hazard. Alternatively, magnetic resonance imaging (MRI) technology recently plays an important role in the field of medical imaging diagnosis. MRI can not only track where the embolic agents are located precisely on real time ${ }^{12-16}$ but also prevent the patient from the radiation hazards. ${ }^{17,18} \mathrm{In}$ the MRI technology, superparamagnetic iron oxide nanoparticles (SPIO NPs) are usually added into embolic agents to endow them with enhanced MRI visuality. For instance, Chung et al prepared chitosan microspheres encapsulated with SPIO NPs using a dripping method. ${ }^{14}$ The obtained chitosan microspheres showed good visuality under MRI. Wang et al reported poly(vinyl alcohol) particles loaded with SPIO NPs and anticancer drug doxorubicin (DOX). ${ }^{19}$ Although these particles also showed good visuality under MRI, the large size $(1.5-2 \mathrm{~mm})$ of the particles is not convenient to inject them through a microcatheter and is not conducive to embolize the blood vessel. Killer et al developed acrylate hydrogel filaments encapsulated with SPIO NPs. ${ }^{20}$ The hydrogel filaments were also not easy to be delivered in a microcatheter. Chio et al reported SPIO NPs-embedded chitosan microspheres prepared by emulsion method. Although the size of the microspheres is relatively small, the wide size distribution (300 500 $\mu \mathrm{m})$ would lead to an uncontrollable embolization. ${ }^{13}$

In the earlier TACE therapy, the embolic agent usually contains single drug, for example, Lipiodol ${ }^{\circledR}$ emulsion loaded with a certain anti-cancer drug. In general, such emulsion has poor stability because the loaded drug diffuses quickly into the blood circulation. Other drug-eluting beads as the TACE materials have been developed, such as DOX-loaded poly(vinyl alcohol) beads and cisplatin-loaded gelatin beads. ${ }^{3,4,7,21-23}$ In comparison with Lipiodol ${ }^{\circledR}$ emulsion, these synthetic or natural polymer microspheres have better stability. Recently, Kim et al prepared calcium alginate (Ca-ALG) microspheres co-encapsulated with SPIO NPs and a single drug (6-methoxyethylamino numonafide), which exhibited good visuality under MRI. The drug release behavior could be modulated by the content of SPIO NPs in the microspheres. ${ }^{24}$ In order to improve the efficacy of TACE and reverse drug resistance of tumor cells, synergistic effect of multi-drug and multi-target therapy may be an imperative. ${ }^{25,26}$

Taking into account the advantage of droplet-based microfluidic technique, for example, size control in the range of tens to hundreds of micrometers and narrow size distribution of microspheres, ${ }^{27,28}$ in this work, Ca-ALG microspheres co-encapsulated with SPIO NPs and dual drugs were prepared via microfluidic technique. Alginate is a kind of natural polyelectrolyte with high charge density and possesses good biocompatibility and stability due to the lack of responding enzyme (ie, alginase) in mammals. SPIO NPs were existed stably in Ca-ALG microspheres, endowing the microspheres with good visuality under MRI. The resultant microspheres can realize the integration of embolotherapy, chemotherapy, and postoperative diagnosis, improving the treatment efficiency of TACE.

\section{Materials and methods Materials}

Sodium alginate (low viscosity, denoted as Na-ALG) and 5-fluorouracil (5-Fu, 99\% in purity) were purchased from Sigma-Aldrich (St Louis, MO, USA). Trisodium citratestabled SPIO NPs were prepared by a method described elsewhere..$^{29,30}$ The diameter of the obtained SPIO NPs was $\sim 10 \mathrm{~nm}$ measured from the image of transmission electron microscopy (TEM). The SPIO NPs were re-dispersed in water at a concentration of $10 \mathrm{mg} / \mathrm{mL}$ as a stock solution for further use. DOX hydrochloride (DOX. $\mathrm{HCl}, 99.3 \%$ in purity) was produced by Beijing Huafeng United Technology Co. Ltd (Beijing, China). All other chemicals were of analytic grade and purchased from Sinopharm Chemical Reagent Co. Ltd (Shanghai, China). Dulbecco's Modified Eagle's Medium (DMEM) and fetal bovine serum (FBS) were purchased from GE Healthcare Life Sciences HyClone Laboratories (Logan, UT, USA). 3-(4,5-Dimethyl-2-thiazolyl)-2,5-diphenyltetrazolium bromide (MTT) was purchased from Sigma-Aldrich (St Louis, MO, USA).

\section{Preparation of SPIO-loaded alginate microspheres}

A T-junction microfluidic device was fabricated according to a method described elsewhere. ${ }^{31,32}$ As reported previously, CaALG microspheres loaded with SPIO NPs (denoted as SPIO/ Ca-ALG) were prepared using this microfluidic device. ${ }^{32}$ Liquid paraffin containing $2 \mathrm{wt} \%$ of span 80 and $0.8 \mathrm{wt} \%$ in situ-formed $\mathrm{CaCl}_{2}$ NPs was adopted as a continuous phase prepared using an emulsion process and followed by solvent evaporation. ${ }^{32,33} \mathrm{~A}$ calculated amount of $\mathrm{CaCl}_{2}$ powder was first dissolved in ethanol and then mixed with liquid paraffin containing $2 \mathrm{wt} \%$ of span 80 to form an emulsion under sonication. Then ethanol in the emulsion was evaporated by heating to $60^{\circ} \mathrm{C}$ overnight and a clear liquid paraffin solution 
containing in situ-formed $\mathrm{CaCl}_{2}$ NPs was obtained. The diameter of $\mathrm{CaCl}_{2}$ NPs is $\sim 11 \mathrm{~nm}$ with polydispersion index of 0.22 for the case of $0.8 \mathrm{wt} \% \mathrm{CaCl}_{2}$ in liquid paraffin measured by dynamic light scattering. ${ }^{32}$ The aqueous solution of Na-ALG $(2 \mathrm{wt} \%)$ containing varied amount of SPIO NPs $(0-6.0 \mathrm{mg} / \mathrm{mL})$ was used as a dispersed phase. Both continuous and dispersed phases were separately pumped into the microchannels using digitally controlled syringe pumps (NE-1000; New Era Pump Systems, Inc, Farmingdale, NY, USA). Na-ALG droplets containing SPIO NPs were produced and pre-crosslinked by $\mathrm{CaCl}_{2} \mathrm{NPs}$ in the microchannel. Subsequently, these droplets dripped into an aqueous solution of $\mathrm{CaCl}_{2}(0.2 \mathrm{~mol} / \mathrm{L})$ and were maintained in the solution for several hours for further crosslinking. The resultant SPIO/Ca-ALG microspheres were collected using a magnet and purified by washing several times with alcohol and water. The purified SPIO/Ca-ALG microspheres were dispersed in water for further characterization. The size of SPIO/Ca-ALG microspheres can be modulated by changing the flow rate ratio of the continuous phase and the dispersed phase.

\section{Characterization of SPIO/Ca-ALG microspheres}

The morphologies of the wet SPIO/Ca-ALG microspheres were characterized by an inverted optical microscope (DSY5000X; Chongqing COIC Industrial Co. Ltd., Chongqing, China). The average size and length-diameter $(\mathrm{L} / \mathrm{D})$ ratio of the microspheres were evaluated using Image Pro Plus software (Roper Technologies, Sarasota, FL, USA). The frozen dried microspheres were coated with gold and then imaged on an environmental scanning electron microscope (ESEM, Quanta 200; FEI, Eindhoven, the Netherlands) at an accelerating voltage of $20 \mathrm{kV}$. The elemental composition of the microspheres was analyzed using SEM combined with energy dispersed X-ray spectrometer system (SEMEDX, Genesis; EDAX Inc., Eindhoven, the Netherlands). Fourier transform infrared spectra (FT-IR) of SPIO NPs and microspheres were recorded on a Fourier transform infrared spectrometer (equinox 55; Bruker, Billerica, MA, USA) using $\mathrm{KBr}$ pellet method.

The content of Fe in the SPIO/Ca-ALG microspheres was determined on a flame atomic absorption spectrometer (AAS, AA-300; Perkin Elmer, Waltham, MA, USA). The standard curve of iron was made based on the absorption values of a series of iron solutions with the concentration of $1-5 \mu \mathrm{g} / \mathrm{mL}$ at the wavelength of $248.3 \mathrm{~nm}$. The slit width was $0.2 \mathrm{~nm}$ and the current of iron hollow cathode lamp was set as $10 \mathrm{~mA}$. The resulted standard curve equation was $\mathrm{A}=0.0232 \mathrm{C}-0.0005$ with correlation coefficient $R^{2}=0.9996$. The samples were made by dissolving microspheres in a mixed solution of concentrated nitric acid and concentrated hydrochloric acid $(1 / 1$ of $\mathrm{v} / \mathrm{v})$, and then volumed to $100 \mathrm{~mL}$. The atomic absorption signal was measured under the conditions described earlier.

The hysteresis loops of the SPIO/Ca-ALG microspheres were tested on a vibrating sample magnetometer (VSM 7404; Lakeshore, Westerville, OH, USA) with a scanning range from $-15,000$ Oe to 15,000 Oe at room temperature. The crystal structure of SPIO NPs was measured on a powder X-ray diffraction instrument (XRD, X'Pert PRO; PANalytical B.V., Almelo, the Netherlands) with $2 \theta$ ranging from $20.0^{\circ}$ to $80.0^{\circ}$ in a step of $0.017^{\circ}$ under a $\mathrm{Cu} \mathrm{K} \alpha$ radiation. The operation voltage was $40 \mathrm{kV}$ and the current was $40 \mathrm{~mA}$.

\section{MRI of SPIO/Ca-ALG microspheres in vitro}

In a plastic test tube with specified amount of agarose gel (1 wt\%), small amount of SPIO/Ca-ALG microspheres was uniformly added on the surface of agarose gel. Subsequently, the formed layer of the microspheres was covered by small amount of hot agarose solution ( $1 \mathrm{wt} \%)$. After cooling to room temperature, the gel sample was imaged on MR scanner (Discovery MR750w 3.0 Tesla; GE Healthcare, Milwaukee, WI, USA). The Ca-ALG microspheres without SPIO NPs were used as a reference. FSE-XL sequence was used for T2-weighted spin-echo imaging. Relevant parameters are repetition time $3,000 \mathrm{~ms}$, field of view $130 \times 130 \mathrm{~mm}$, and echo time $81.4 \mathrm{~ms}$.

\section{Loading and in vitro release of drugs in SPIO/Ca-ALG microspheres}

$\mathrm{DOX} \cdot \mathrm{HCl}$ and $5-\mathrm{Fu}$ were used as model drugs in this study. Drug-loaded SPIO/Ca-ALG microspheres were prepared using swelling adsorption method. Briefly, weighted freezedried microspheres $(\sim 50 \mathrm{mg})$ were immersed in $3 \mathrm{~mL}$ of $5-\mathrm{Fu}(10 \mathrm{mg} / \mathrm{mL})$ and DOX. $\mathrm{HCl}(1.38 \mathrm{mg} / \mathrm{mL})$ solution. The mixture was placed on an oscillator with a rate of $100 \mathrm{rpm}$ at $37^{\circ} \mathrm{C}$ for 48 hours. After centrifugation and washing with water, the samples were dried in oven for further use. The solution mixture of the supernatant of centrifugation and the washing liquid were added with water to a constant volume. The concentrations of $5-\mathrm{Fu}$ and $\mathrm{DOX} \cdot \mathrm{HCl}$ in the solution mixture were quantified by using a UV-visible spectrophotometer (UV-2550; Shimadzu, Kyoto, Japan) at the wavelength of 265 and $479 \mathrm{~nm}$, respectively. All the procedures that involved DOX $\cdot \mathrm{HCl}$ were performed in the dark. Drug loading capacity (LC) and encapsulation efficiency 
(EE) of the microspheres were determined by using the following equations:

$$
\begin{aligned}
\mathrm{LC}(\%)= & {[(\text { Amount of drug in microspheres }) /} \\
& (\text { Amount of drug-loaded } \\
& \text { microspheres })] \times 100
\end{aligned}
$$

$\mathrm{EE}(\%)=[($ Amount of drug in microspheres $) /$ (Amount of feeding drug) $] \times 100$

In vitro release profiles of drugs from the microspheres were performed by a dialysis method. Briefly, the weighted freeze-dried drug-loaded microspheres $(\sim 10 \mathrm{mg})$ were placed in a dialysis bag (the cutoff molecular weight was $14,000 \mathrm{Da}$ ) and $1 \mathrm{~mL}$ phosphate-buffered solution (PBS, $\mathrm{pH} 7.4,0.01 \mathrm{~mol} / \mathrm{L})$ was added. The dialysis bag was immersed in $19 \mathrm{~mL}$ PBS in a capped flask and placed on an oscillator with a rate of $100 \mathrm{rpm}$ at $37^{\circ} \mathrm{C}$. At a designed time interval, $4 \mathrm{~mL}$ of the release medium was removed and replaced with an equal volume of fresh medium. The amount of $5-\mathrm{Fu}$ and $\mathrm{DOX} \cdot \mathrm{HCl}$ released was quantified by UV-vis spectrophotometer at the wavelength 265 and $479 \mathrm{~nm}$, respectively. The experiments were performed in triplicate.

\section{In vitro cytotoxicity of SPIO/Ca-ALG microspheres}

Liver hepatocellular cells (HepG2 cells) were used for in vitro cytotoxicity evaluations of the drug-loaded SPIO/Ca-ALG microspheres and free drugs. The microspheres were sterilized with UV light radiation for 15 minutes. In a $1.5-\mathrm{mL}$
Eppendorf tube, the microspheres suspension $(8 \mathrm{mg} / \mathrm{mL})$ or free drug solution $(500 \mu \mathrm{g} / \mathrm{mL})$ was mixed with DMEM. The mixture was incubated at $37^{\circ} \mathrm{C}$ for 24 hours. HepG2 cells were seeded at $8 \times 10^{3}$ cells/well in a 96-well cell culture plate with DMEM containing 10\% FBS overnight. Then the medium for cell culture was removed and replaced with $200 \mu \mathrm{L}$ of the supernatant of the microspheres incubation system. The supernatant and cells were then incubated at $37^{\circ} \mathrm{C}$ in $5 \% \mathrm{CO}_{2} 24$ hours. Subsequently, $20 \mu \mathrm{L}$ of 3-(4,5dimethylthiazol-2-yl)-2,5-diphenyl tetrazolium bromide (MTT) solution ( $5 \mathrm{mg} / \mathrm{mL})$ was added to the cells in each well. After 4 hours of incubation at $37^{\circ} \mathrm{C}$, the medium containing MTT was removed and replaced by $150 \mu \mathrm{L}$ of dimethylsulfoxide to dissolve the formazan crystals formed by living cells. Absorbance was measured at $490 \mathrm{~nm}$ by using an iEMS microplate reader (Labsystems, Helsinki, Finland). All the experiments were repeated four times.

\section{Statistical analysis}

All the data were presented as mean \pm standard deviation (SD). Student's $t$-test was used to evaluate the significance in two groups. $P<0.05$ and $P<0.01$ were considered as significant difference.

\section{Results and discussion Preparation and characterization of SPIO-loaded alginate microspheres}

Figure 1A schematically illustrates the preparation process of SPIO/Ca-ALG microspheres using microfluidics technique.
A

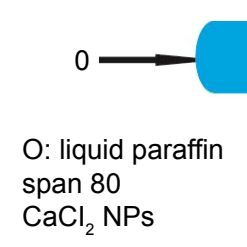

Pre-crosslinked droplet

SPIO/Ca-ALG microsphere

- SPIO NPS
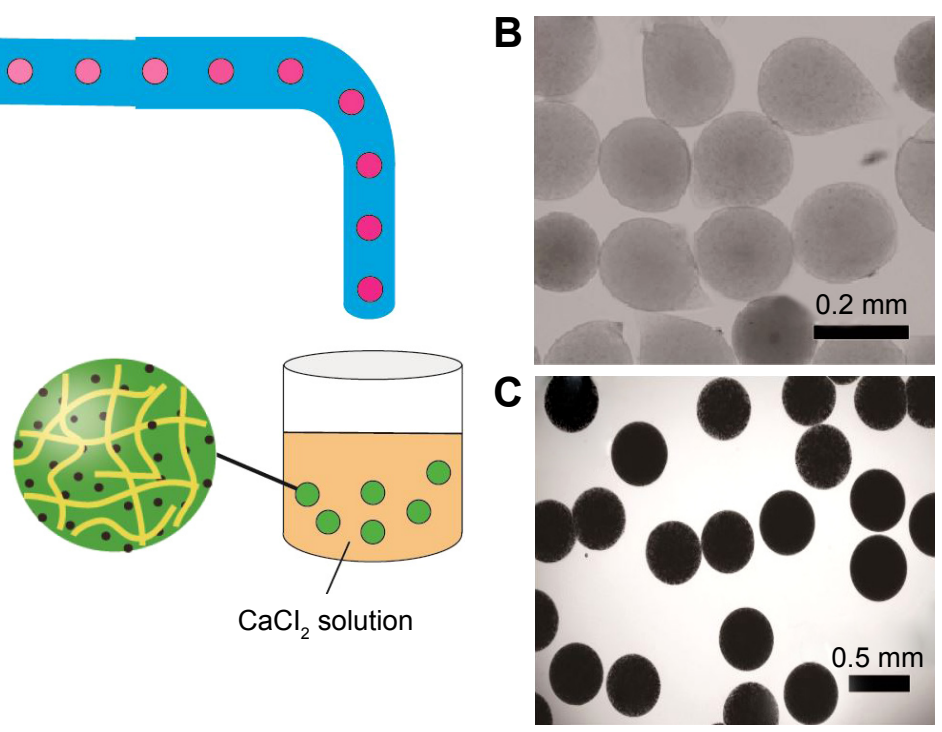

Figure I Schematic illustration of the preparation of the microspheres and their optical photographs.

Notes: (A) Schematic illustration of SPIO/Ca-ALG microspheres prepared using a T-junction microfluidic device with Na-ALG aqueous solution containing various amount of SPIO NPs as a dispersed phase and liquid paraffin containing $\mathrm{CaCl}_{2}$ NPs and span 80 as a continuous phase. (B) The optical photographs of Ca-ALG microspheres without SPIO NPs. (C) The optical photographs of Ca-ALG microspheres encapsulated with $3 \mathrm{mg} / \mathrm{mL}$ SPIO NPs.

Abbreviations: O, oil phase; W, aqueous phase; SPIO, superparamagnetic iron oxide; Ca-ALG, calcium alginate; Na-ALG, sodium alginate; NPs, nanoparticles. 
Aqueous solution of Na-ALG containing SPIO NPS was used as the dispersed phase (aqueous phase, W) and liquid paraffin containing span 80 and $\mathrm{CaCl}_{2}$ NPs was used as continuous phase (oil phase, $\mathrm{O}$ ). Both the phases were separately pumped into the microchannels of the T-junction microfluidic device. At the orifice of the two phases, the stream of aqueous phase was broken off as discrete droplets due to the shearing force of the oil phase and the different interfacial tension between the two phases. In addition, $\mathrm{CaCl}_{2} \mathrm{NPs}$ in the oil phase were gradually moved to the interface of droplets due to a hydrophilic interaction, leading to an ionic crosslinking between $\mathrm{Ca}^{2+}$ and carboxyl groups of alginate. Because of the low concentration of $\mathrm{CaCl}_{2}$ in the oil phase and limited diffusion, the ionic crosslinking may occur on the surface of the droplets. Nevertheless, this pre-crosslinking can increase the rigidity of droplets, avoiding droplet deformation caused by the gravitational force and interfacial effect when they dripped into the collection solution. ${ }^{32}$ Also, this pre-crosslinking is beneficial to maintain the regular spherical shape of droplets. ${ }^{34,35}$ When the droplets dripped into the collection solution of $\mathrm{CaCl}_{2}$, a sufficient crosslinking can be achieved by $\mathrm{Ca}^{2+}$ ions diffusion and penetration. Figure 1B shows the optical photograph of the Ca-ALG microspheres without SPIO NPs. The size of microspheres was found to be $\sim 200 \mu \mathrm{m}$. Figure $1 \mathrm{C}$ shows the photograph of SPIO/Ca-ALG microspheres with the diameter of $\sim 600 \mu \mathrm{m}$. Both the types of microspheres show regular spherical shape and uniform size. The size of the Ca-ALG microspheres with/without SPIO NPs can be effectively controlled by adjusting flow ratio of both phases and the dimension of the microfluidic device as previously reported. ${ }^{32}$ As shown in Figure 1C and S1 (Supplementary materials), the content of SPIO NPs in Ca-ALG microspheres does not affect the spherical shape and size of the microspheres. But the color of the microspheres became dark with the increase of SPIO NP content due to the inherent nonpenetration of light into SPIO. Their size, size distribution, and length and diameter ratio (L/D) are listed in Table $\mathrm{S} 1$.

Figure 2A-C shows SEM images of Ca-ALG microspheres prepared in the presence of $0,3.0$ and $6.0 \mathrm{mg} / \mathrm{mL}$ of SPIO NPs in the aqueous phase. The typical spherical shape and uniform size $(\sim 400 \mu \mathrm{m})$ can be clearly observed. Figure 2A1-C1 shows partial enlarged SEM images of the corresponding microsphere surface. The surface of Ca-ALG microspheres without SPIO NPs looks smooth and there are no $\mathrm{CaCl}_{2} \mathrm{NPs}$ or its aggregation (Figure 2A1). These residual soluble calcium salts should have been cleaned up during the purifying process of microspheres by using alcohol and water before SEM imaging. But the surface of SPIO/Ca-ALG microspheres look rather rough and number of small white particles can be observed on their surfaces (Figure 2B1). Furthermore, the amount of the white particles increases with an increase of the SPIO NP content in the microspheres (Figure 2C1). These white particles should be SPIO NPs encapsulated in the microspheres. In order to verify this inference, the area indicated by red circle in Figure 2B1 was chosen to analyze by EDX. As shown in Figure 2D, the absorption peak of Fe element appeared at $6.4 \mathrm{Kev}$. The atomic weight percent of $\mathrm{Fe}$ in the selected area was found to be $7.5 \%$. This result confirms that SPIO NPs encapsulated in $\mathrm{Ca}-\mathrm{ALG}$ microspheres. In addition, the stable dispersion of SPIO NPs in the microspheres may be attributed to the interaction between SPIO NPs and carboxyl and hydroxyl groups in Ca-ALG microspheres. This can be verified by the FT-IR spectra of the microspheres (Figure S2).
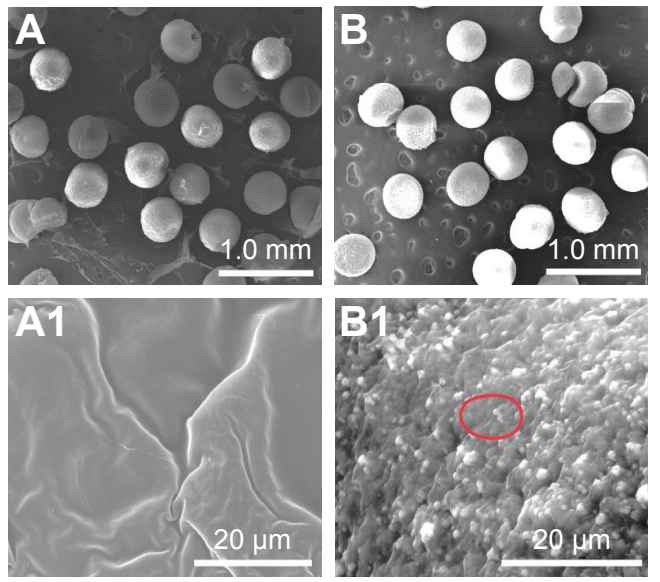
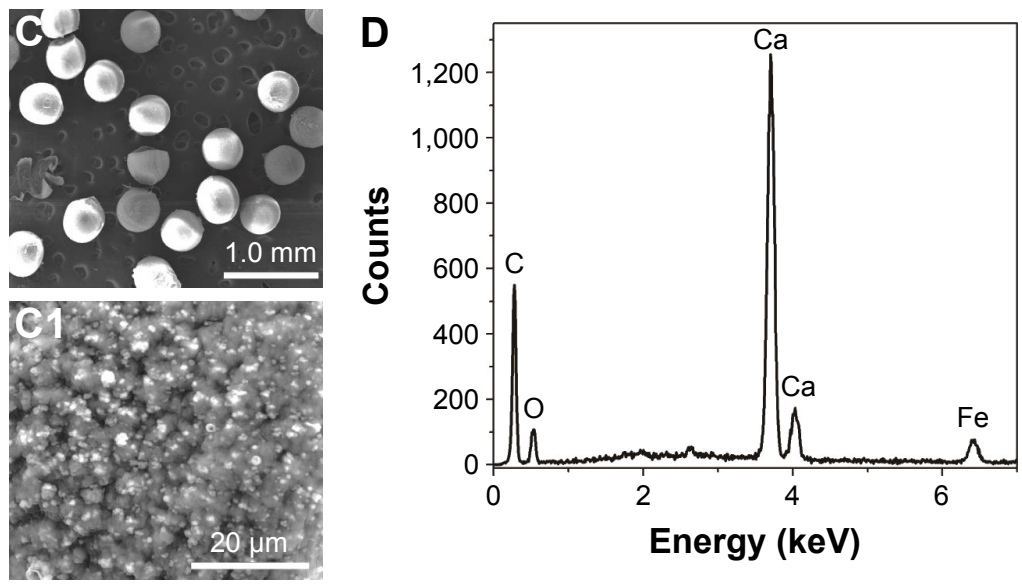

Figure 2 SEM images and EDX spectra of SPIO/Ca-ALG microspheres.

Notes: (A-C) SEM images of SPIO/Ca-ALG microspheres prepared with 0, 3.0, and $6.0 \mathrm{mg} / \mathrm{mL}$ SPIO NPs, respectively. (AI-CI) Enlarged SEM image of the surface of one microsphere in A-C, respectively. (D) EDX spectra corresponding to the area marked by the red circle in Figure $\mathbf{B} \mathbf{I}$, indicating the existence of element Fe. Abbreviations: SEM, scanning electron microscope; EDX, energy dispersed X-ray spectrometer system; SPIO, superparamagnetic iron oxides; Ca-ALG, calcium alginate. 
Alternatively, the amount of Fe in SPIO/Ca-ALG microspheres can also be determined by using an atomic absorption spectrometer. For instance, the Fe content in SPIO NPs stock solution $(10 \mathrm{mg} / \mathrm{mL})$ is $4.298 \mathrm{mg} / \mathrm{mL}$, which is equal to $5.924 \mathrm{mg} / \mathrm{mL}$ of $\mathrm{Fe}_{3} \mathrm{O}_{4}$. Thus, in theory, the content of $\mathrm{Fe}$ in various dried $\mathrm{SPIO} / \mathrm{Ca}-\mathrm{ALG}$ microspheres should be 1.3, $12.7,59.0$, and $109.3 \mathrm{mg} / \mathrm{g}$ in the case of the microspheres prepared in the presence of $0.06,0.6,3.0$, and $6.0 \mathrm{mg} / \mathrm{mL}$ SPIO NPs. In these experiments, the corresponding measured values were found to be $1.34,14.33,53.78$, and $74.97 \mathrm{mg} / \mathrm{g}$. The data were quite close to the theoretical values, suggesting an effective encapsulation of SPIO NPs in the microspheres and less leakage during purification.

As used for MRI-visible materials, an essential property of SPIO/Ca-ALG microspheres is their magnetic response. Figure 3A-D shows that SPIO NPs or SPIO/ $\mathrm{Ca}-\mathrm{ALG}$ microspheres can be dispersed in water to form a homogeneous suspension. But they were completely separated from the suspension in the presence of external magnetic field. This result visually indicated that both SPIO NPs and SPIO/Ca-ALG microspheres possess excellent magnetic response.

In order to further confirm the superparamagnetic property of the SPIO NPs and SPIO/Ca-ALG microspheres, their magnetic hysteresis loops were measured by vibrating sample magnetometer as shown in Figure 3E. The saturation magnetic moment of SPIO NP suspension was found to be $69 \mathrm{emu} / \mathrm{g}$ (curve 1). By contrast, those values of Ca-ALG microspheres containing 0.6 and $6 \mathrm{mg} / \mathrm{mL}$ of SPIO NPs were rather low (curve 2 and 3), which should be attributed to the low content of SPIO NPs. Nevertheless, the magnetization curves of three samples were symmetrical and passed through the origin with no hysteresis, suggesting that the magnetic SPIO NPs and the consequent magnetic SPIO/Ca-ALG microspheres are typical paramagnetic materials. ${ }^{36}$ The XRD patterns of SPIO NPs and two samples of SPIO/Ca-ALG microspheres are quite similar as shown in Figure $3 \mathrm{~F}$. The distinct diffraction peaks can be observed at $2 \theta$ of 29.84 , $35.27,43.04,53.31,56.82$, and 62.56 , those correspond to the crystal plane of (220), (311), (400), (422), (511), and (440)
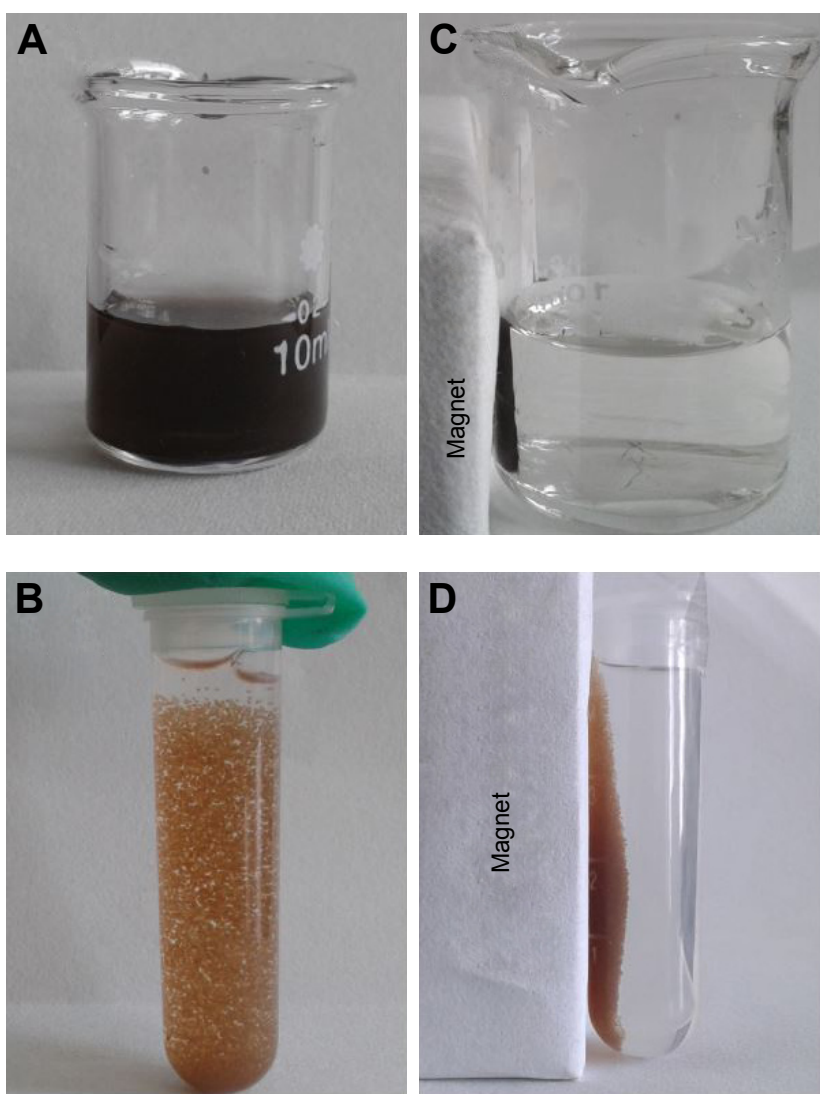

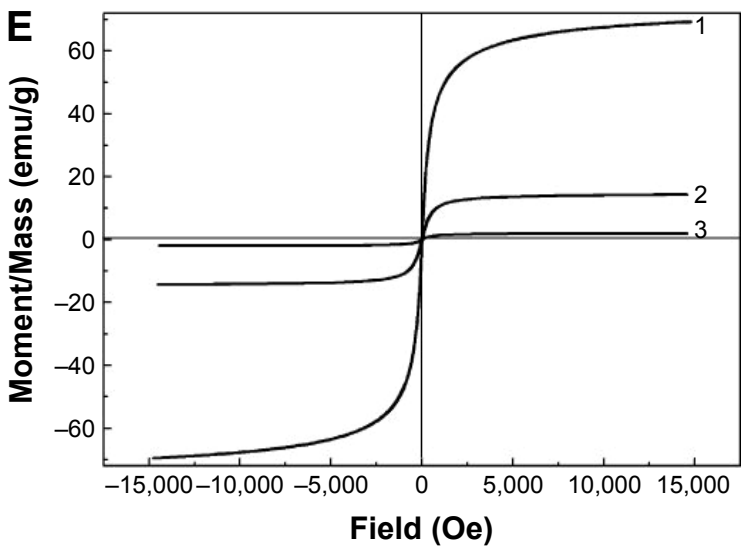

$\mathbf{F}$

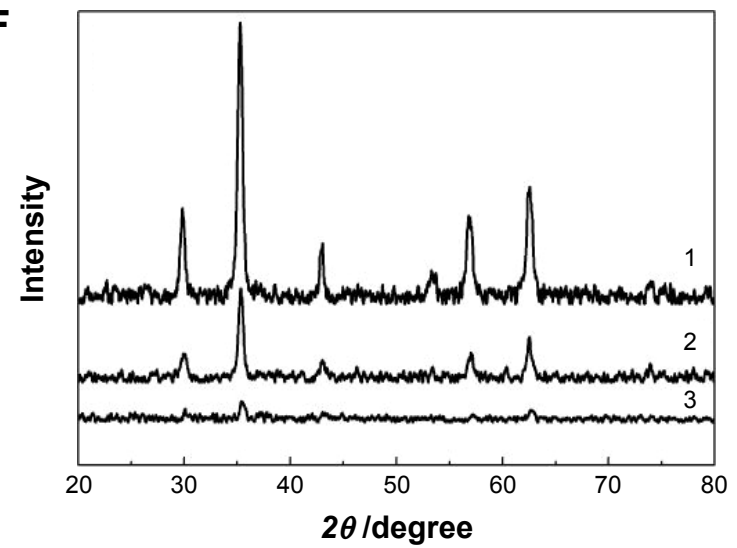

Figure 3 Magnetic properties of SPIO NPs and SPIO/Ca-ALG microspheres.

Notes: (A and B) Photographs of SPIO NPs suspension and SPIO/Ca-ALG microspheres suspension in the absence of magnet. (C and D) Photographs of SPIO NPs suspension and SPIO/Ca-ALG microspheres in the presence of a magnet. (E) Hysteresis loops and (F) X-ray diffraction patterns of SPIO NPs suspension (IO mg/mL, curve I), SPIO/Ca-ALG microspheres suspension containing $6.0 \mathrm{mg} / \mathrm{mL} \mathrm{SPIO} \mathrm{(curve} \mathrm{2),} \mathrm{and} 0.6 \mathrm{mg} / \mathrm{mL}$ SPIO (curve 3), respectively.

Abbreviations: SPIO, superparamagnetic iron oxides; Ca-ALG, calcium alginate. 


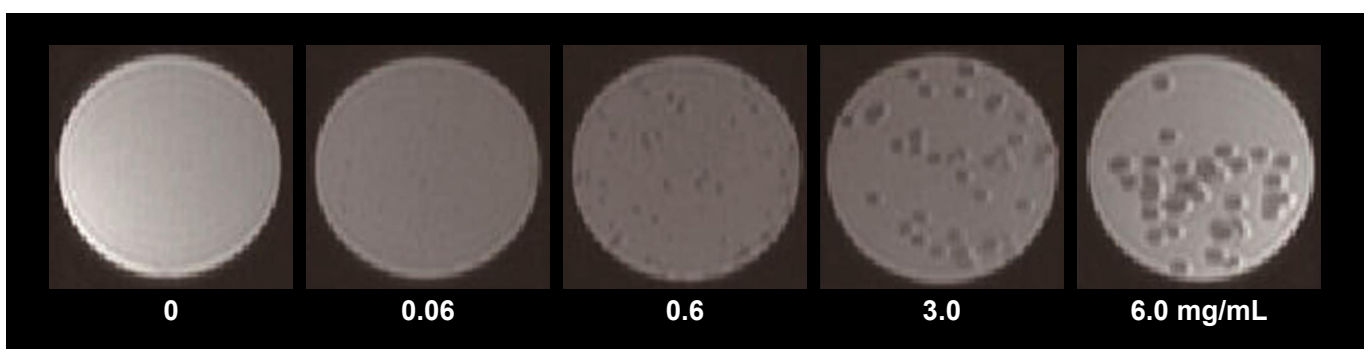

Figure 4 MR images of SPIO/Ca-ALG microspheres $(\sim 500 \mu \mathrm{m})$ with varied content of SPIO NPs.

Abbreviations: MR, magnetic resonance; SPIO, superparamagnetic iron oxides; Ca-ALG, calcium alginate; NPs, nanoparticles.

of inverse spinel type $\mathrm{Fe}_{3} \mathrm{O}_{4}$ with face-centered cubic phase, respectively. The spectra are also consistent with the standard characteristic diffraction peaks of $\mathrm{Fe}_{3} \mathrm{O}_{4}$ (JCPDS card no 19-0619, magnetite crystal). It is noted that the diffraction intensities of two samples of SPIO/Ca-ALG microspheres were relatively weak because the low content of SPIO NPs that were encapsulated.

\section{MR images of SPIO/Ca-ALG microspheres in vitro}

Figure 4 shows the T2-weighted MR images of the Ca-ALG microspheres $(\sim 500 \mu \mathrm{m})$ containing varied content of SPIO NPs (from 0 to $6.0 \mathrm{mg} / \mathrm{mL}$ ). As described in the "Materials and methods" section, SPIO/Ca-ALG microspheres were embedded in agarose gel in a plastic test tube (gray round plate in Figure 4). Ca-ALG microspheres can be gradually detected with an increase of SPIO NP content in the microspheres. However, overmuch SPIO in the microspheres would enlarge the size of the microspheres. The other MR images of SPIO/Ca-ALG microspheres with varied size showed similar results (Figure S3). Considering the fact that microspheres containing $0.6 \mathrm{mg} / \mathrm{mL}$ SPIO NPs can be clearly detected by MRI, they will be used for the subsequent study of drug loading, drug release, and their cytotoxicity.

\section{Drug loading and in vitro release of SPIO/ Ca-ALG microspheres}

As anticancer drugs, DOX. $\mathrm{HCl}$ and 5-Fu are commonly used clinically. Usually, a combination of DOX. $\mathrm{HCl}$ and $5-\mathrm{Fu}$ is used to enhance the treatment efficiency. In this work, SPIO/ Ca-ALG microspheres loaded with dual drugs (DOX.HCl and 5-Fu) were prepared by swelling adsorption method. As a control, two kinds of drugs were also loaded in the Ca-ALG microspheres without SPIO NPs. Table 1 lists their drug LC and EE. It was found that two carriers exhibit higher $\mathrm{LC}$ and $\mathrm{EE}$ for $\mathrm{DOX} \cdot \mathrm{HCl}$ rather than $5-\mathrm{Fu}$. It may be ascribed to a strong electrostatic interaction between $\mathrm{DOX} \cdot \mathrm{HCl}$ with positive charge and carboxyl groups of Ca-ALG with negative charge. ${ }^{30}$ In contrast, there is only hydrogen bond between 5 -Fu and alginate with relatively weak interaction force. The interaction between the two drugs and alginate could be deduced from their chemical structure as shown in Figure S4. In addition, the results of Table 1 indicated that both carriers do not show significant difference of loading ability for same drug. In other words, the presence of SPIO NPs does not affect the drug loading ability of Ca-ALG microspheres.

Figure 5 shows in vitro cumulative release curves of $\mathrm{DOX} \cdot \mathrm{HCl}$ (Figure 5A) and 5-Fu (Figure 5B) from the SPIO/Ca-ALG and Ca-ALG microspheres, respectively. It was found that both the carriers exhibit typical slow and sustained drug release profiles. For instance, the cumulative release percentages of DOX $\cdot \mathrm{HCl}$ and $5-\mathrm{Fu}$ from the $\mathrm{SPIO} /$ Ca-ALG microspheres were found to be $28 \%$ and $33 \%$ within 7 days, respectively. In contrast, these cumulative release percentages were slightly lower than that from the Ca-ALG microspheres. The reason may be attributed to the fact that SPIO NPs trapped in the network of microspheres occupied a certain space, resulting in slow diffusion and release of drug molecules. In addition, the release rate of 5-Fu from both the microspheres is slightly higher than that of DOX $\cdot \mathrm{HCl}$. This may be ascribed to the differences of molecular weight of both the drugs. The molecular weight of $5-\mathrm{Fu}$ is $130.08 \mathrm{~g} / \mathrm{mol}$, whereas the molecular weight of DOX $\cdot \mathrm{HCl}$ is $543.52 \mathrm{~g} / \mathrm{mol}$. Small molecules are conducive to their diffusion in the matrix of Ca-ALG microspheres. Furthermore, another reason

Table I LC and EE of dual drugs in SPIO/Ca-ALG and Ca-ALG microspheres

\begin{tabular}{llllll}
\hline & Microspheres & \multicolumn{2}{c}{ Ca-ALG } & & \multicolumn{2}{c}{ SPIO/Ca-ALG } \\
\cline { 2 - 3 } Drug & LC (\%) & EE (\%) & & LC (\%) & EE (\%) \\
\hline DOX.HCl & $6.1 \pm 0.1$ & $60.8 \pm 3.5$ & & $5.7 \pm 0.4$ & $62.9 \pm 2.3$ \\
$5-\mathrm{Fu}$ & $1.8 \pm 0.1$ & $2.7 \pm 0.2$ & & $1.6 \pm 0.1$ & $2.6 \pm 0.2$ \\
\hline
\end{tabular}

Notes: Data were presented as mean \pm standard deviation.

Abbreviations: LC, loading capacity; EE, encapsulation efficiency; Ca-ALG, calcium alginate; SPIO, superparamagnetic iron oxides; DOX.HCl, doxorubicin hydrochloride; 5-Fu, 5-fluorouracil. 

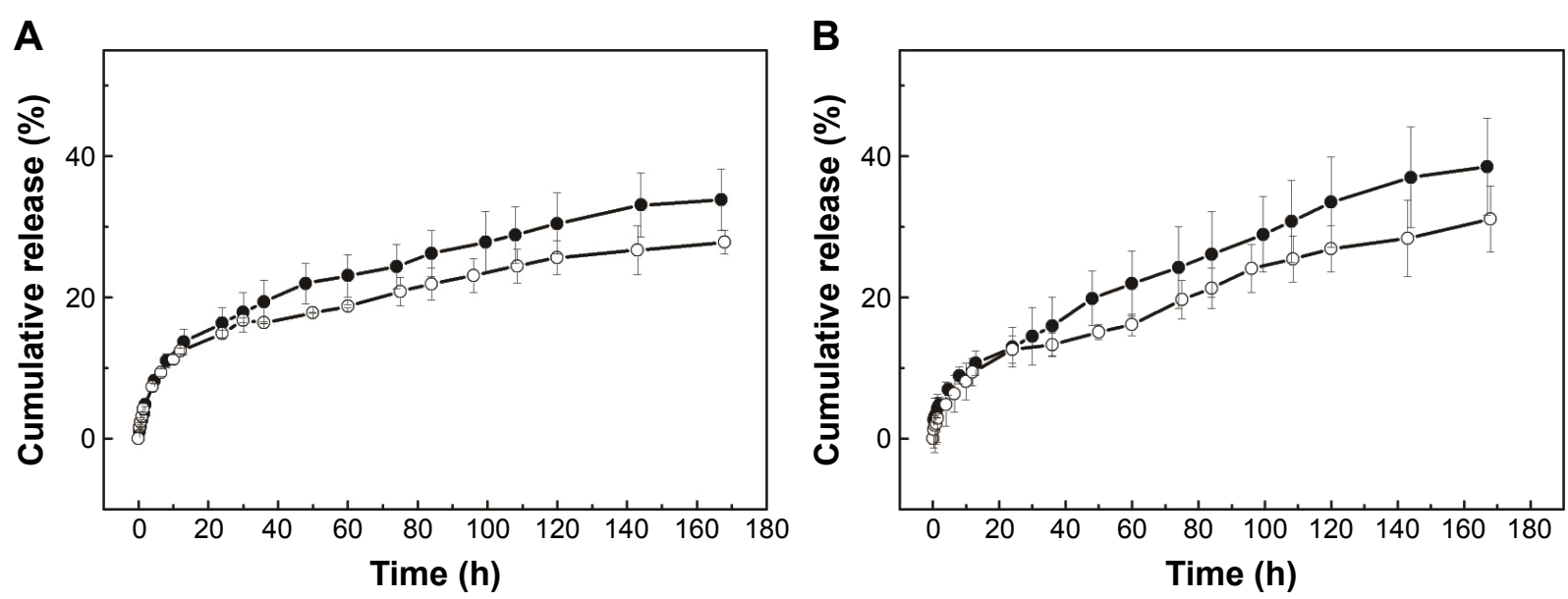

Figure 5 Drug release profiles of dual-drug-loaded microspheres at $37^{\circ} \mathrm{C}$ in PBS ( $n=3$; mean \pm standard deviation).

Notes: (A) DOX.HCl; (B) 5-Fu. Solid circles: Ca-ALG microspheres. Hollow circles: SPIO/Ca-ALG microspheres.

Abbreviations: DOX.HCl, doxorubicin hydrochloride; 5-Fu, 5-fluorouracil; SPIO, superparamagnetic iron oxides; Ca-ALG, calcium alginate; PBS, phosphate-buffered solution.

may involve the strong electrostatic interaction between $\mathrm{Ca}-\mathrm{ALG}$ and DOX.HCl as mentioned above. Nevertheless, such slow and sustained drug release behavior of the dual drugs from SPIO/Ca-ALG microspheres is beneficial to TACE therapy.

To illustrate the possible mechanism of the drug release from the microspheres, zero-order equation, first-order equation, Higuchi's equation, and Korsmeyer-Peppas model (the Power Law) were used to fit the data of the drug release in Figure 5, respectively. Among all these models used, Korsmeyer-Peppas model is best fitting with all the power exponent $n$ values between 0.43 and 0.53 with relative high correlation coefficient $\left(R^{2}\right)$ and low relative standard derivation value (data in detail not shown). Therefore, the drugs released from these swollen Ca-ALG microspheres are mainly controlled by diffusion within testing time. ${ }^{37}$

\section{In vitro cytotoxicity of SPIO/Ca-ALG microspheres}

HepG2 cells, a kind of human liver hepatocellular cells, were used in this work for in vitro cytotoxicity evaluations. Figure 6 shows corresponding cell viabilities in the presence of dual-drug-loaded Ca-ALG and SPIO/Ca-ALG microspheres. As a control, the cytotoxicity of the free dual drugs $(5-\mathrm{Fu}$ and $\mathrm{DOX} \cdot \mathrm{HCl})$ was also tested. It was found that the dual-drug-loaded microspheres show significant cytotoxicity to HepG2 cells in comparison with that of the microspheres without drugs. For instance, the cell viability of drug-unloaded SPIO/Ca-ALG microspheres was found to be $93 \%$, implying almost nontoxicity to HepG2 cells. In contrast, cell viability in the presence of dual-drug-loaded $\mathrm{SPIO} / \mathrm{Ca}-\mathrm{ALG}$ microspheres decreases significantly to $36 \%$.
It is indicative that most of HepG2 cells were killed by the drugs released from the dual-drug-loaded microspheres. It is noted that the cytotoxicity of the dual-drug-loaded Ca-ALG microspheres is slightly larger than that of SPIO/Ca-ALG loaded with same drugs without significant difference. This may be attributed to relatively slow drug release rate from $\mathrm{SPIO} / \mathrm{Ca}-\mathrm{ALG}$ microspheres. This result is consistent with the above discussion of the drug LC and drug release rate.

\section{Conclusion}

Ca-ALG microspheres encapsulated with SPIO NPs (SPIO/ Ca-ALG) were prepared using droplet-based microfluidic devices. The resultant microspheres showed uniform size and regular spherical shape confirmed by optical photographs

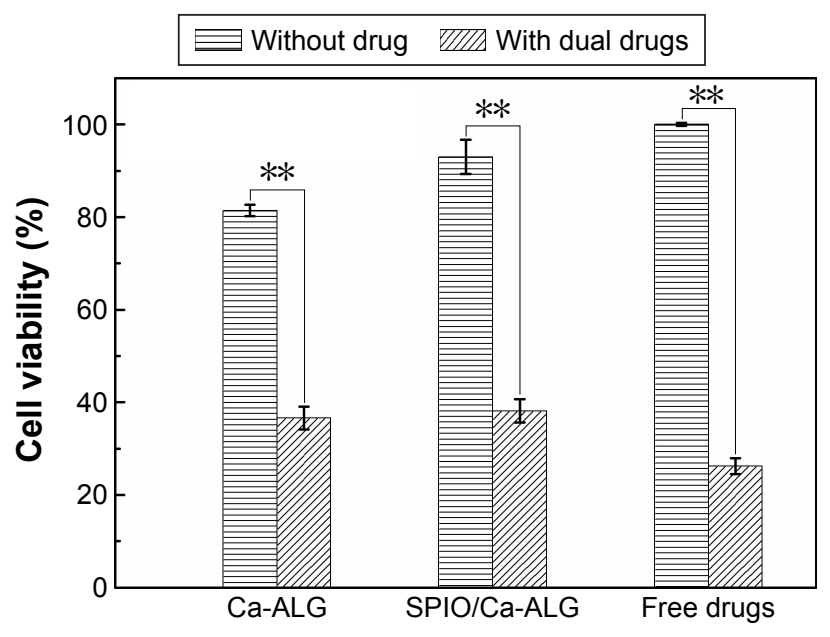

Figure 6 Cell viability of HepG2 cells incubated with various microspheres and free drugs for 24 hours.

Note: $* * P<0.01$ with significant difference.

Abbreviations: SPIO, superparamagnetic iron oxides; Ca-ALG, calcium alginate. 
and SEM images. The SPIO/Ca-ALG microspheres also possessed superparamagnetic property. It was found that the microspheres showed excellent MR visuality in the presence of only $0.6 \mathrm{mg} / \mathrm{mL}$ of SPIO NPs. Two kinds of anticancer drugs (5-Fu and DOX $\cdot \mathrm{HCl}$ ) were loaded in the microspheres simultaneously. The dual-drug-loaded microspheres showed typical sustained release profiles. The reason may be attributed to the restricted drug diffusion due to the hindering of SPIO NPs in the matrix of microspheres. The study of in vitro cytotoxicity indicated that dual-drug-loaded SPIO/ Ca-ALG microspheres show distinct cytotoxicity to HepG2 cells. The prepared SPIO/Ca-ALG microspheres possess dual advantage of excellent MR-visuality and multi-drug chemoembolization.

\section{Acknowledgments}

This work was financially supported by the National Natural Science Foundation of China (51473057) and the National Basic Research Program of China (2012CB932500). The authors thank the Analytical and Testing Center of Huazhong University of Science and Technology, School of Chemistry and Chemical Engineering, Huazhong University of Science and Technology for the related measurements.

\section{Disclosure}

The author reports no conflicts of interest in this work.

\section{References}

1. Chan SPL, Yeo W. Targeted therapy of hepatocellular carcinoma: present and future. J Gastroenterol Hepatol. 2012;27:862-872.

2. Muller A, Rouviere O. Renal artery embolization-indications, technical approaches and outcomes. Nat Rev Nephrol. 2015;11:288-301.

3. Shouval D. Embolization of hepatocellular carcinoma with drug-eluting beads: new insight into the biologic effects of tumor ablation. J Hepatol. 2011;55:1169-1170.

4. Kettenbach J, Stadler A, Von Katzler I, et al. Drug-loaded microspheres for the treatment of liver cancer: review of current results. Cardiovasc Interven Radiol. 2008;31:468-476.

5. Gadaleta CD, Ranieri G. Trans-arterial chemoembolization as a therapy for liver tumors. Crit Rev Oncol Hematol. 2011;80:40-53.

6. Dorenberg EJ, Novakovic Z, Smith HJ, Hafsahl G, Jakobsen JA. Uterine fibroid embolization can still be improved: observations on postprocedure magnetic resonance imaging. Acta Radiologica. 2005;46: $547-553$

7. Huang JY, Kafy S, Dugas A, Valenti D, Tulandi T. Failure of uterine fibroid embolization. Fertil Steril. 2006;85:30-35.

8. Lo CM, Ngan H, Tso WK, et al. Randomized controlled trial of transarterial lipiodol chemoembolization for unresectable hepatocellular carcinoma. Hepatology. 2002;35:1164-1171.

9. Wang Q, Zhang D, Xu HB, Yang X, Shen AQ, Yang Y. Microfluidic one-step fabrication of radiopaque alginate microgels with in situ synthesized barium sulfate nanoparticles. Lab Chip. 2012;12:4781-4786.

10. Wang Q, Qian K, Liu SS, et al. X-ray visible and uniform alginate microspheres loaded with in situ synthesized $\mathrm{BaSO}_{4}$ nanoparticles for in vivo transcatheter arterial embolization. Biomacromol. 2015;16:1240-1246.
11. Wang Q, Zhang D, Yang XL, Xu H, Shen AQ, Yang Y. Atom-economical in situ synthesis of $\mathrm{BaSO}_{4}$ as imaging contrast agents within poly $(\mathrm{N}$ isopropylacrylamide) microgels using one-step droplet microfluidics. Green Chem. 2013;15:2222-2229.

12. Martina MS, Fortin JP, Ménager C, et al. Generation of superparamagnetic liposomes revealed as highly efficient MRI contrast agents for in vivo imaging. $J$ Am Chem Soc. 2005; 127:10676-10685.

13. Choi SY, Kwak BK, Shim HJ, Lee J, Hong SU, Kim KA. MRI traceability of superparamagnetic iron oxide nanoparticle-embedded chitosan microspheres as an embolic material in rabbit uterus. Diagn Interv Radiol. 2015;21:47-53.

14. Chung EY, Kim HM, Lee GH, et al. Design of deformable chitosan microspheres loaded with superparamagnetic iron oxide nanoparticles for embolotherapy detectable by magnetic resonance imaging. Carbohyd Polym. 2012;90:1725-1731.

15. Lee HS, Kim EH, Shao HP, Kwak BK. Synthesis of SPIO-chitosan microspheres for MRI-detectable embolotherapy.J Magn Magn Mater. 2005;293:102-105.

16. Cilliers R, Song Y, Kohlmeir EK, Larson AC, Omary RA, Meade TJ. Modification of embolic-PVA particles with MR contrast agents. Magn Reson Med. 2008;59:898-902.

17. Yuan Z, Ye XD, Dong S, et al. Role of magnetic resonance diffusionweighted imaging in evaluating response after chemoembolization of hepatocellular carcinoma. Eur J Radiol. 2010;75:E9-E14.

18. Yu JS, Kim JH, Chung JJ, Kim KW. Added value of diffusion-weighted imaging in the MRI assessment of perilesional tumor recurrence after chemoembolization of hepatocellular carcinomas. J Magn Reson Imaging. 2009;30:153-160.

19. Wang YXJ, Zhu XM, Liang Q, Cheng CHK, Wang W, Leung KCF. In vivo chemoembolization and magnetic resonance imaging of liver tumors by using iron oxide nanoshell/doxorubicin/poly(vinyl alcohol) hybrid composites. Angew Chem Inter Ed. 2014;53:4812-4815.

20. Killer M, Keeley EM, Cruise GM, Schmitt A, McCoy MR. MR imaging of hydrogel filament embolic devices loaded with superparamagnetic iron oxide or gadolinium. Neuroradiology. 2011;53:449-456.

21. Richardson AJ, Laurence JM, Lam VW. Transarterial chemoembolization with irinotecan in the treatment of colorectal liver metastases: systematic review. J Vasc Interv Radiol. 2013;24:1209-1217.

22. Lewis AL, Dreher MR. Locoregional drug delivery using imageguided intra-arterial drug eluting bead therapy. $J$ Control Release. 2012;161:338-350.

23. van Malenstein H, Maleux G, Vandecaveye V, et al. A randomized phase II study of drug-eluting beads versus transarterial chemoembolization for unresectable hepatocellular carcinoma. Onkologie. 2011;34:368-376.

24. Kim DH, Choy T, Huang S, Green RM, Omary RA, Larson AC. Microfluidic fabrication of 6-methoxyethylamino numonafide-eluting magnetic microspheres. Acta Biomater. 2014;10:742-750.

25. Lane D. Designer combination therapy for cancer. Nat Biotechnol. 2006;24:163-164.

26. Sun TM, Du JZ, Yao YD, et al. Simultaneous delivery of siRNA and paclitaxel via a "two-in-one" micelleplex promotes synergistic tumor suppression. ACS Nano. 2011;5:1483-1494.

27. Ju M, Ji X, Wang C, Shen R, Zhang L. Preparation of solid, hollow, hole-shell and asymmetric silica microspheres by microfluidic-assisted solvent extraction process. Chem Eng J. 2014;250:112-118.

28. Xu S, Nie Z, Seo M, et al. Generation of monodisperse particles by using microfluidics: control over size, shape, and composition. Angew Chem Int Ed Engl. 2005;44:724-728.

29. Hao R, Xing R, Xu Z, Hou Y, Gao S, Sun S. Synthesis, functionalization, and biomedical applications of multifunctional magnetic nanoparticles. Adv Mater. 2010;22:2729-2742.

30. Liu JW, Zhang Y, Wang CY, Xu R, Chen Z, Gu N. Magnetically sensitive alginate-templated polyelectrolyte multilayer microcapsules for controlled release of doxorubicin. J Phys Chem C. 2010;114:7673-7679.

31. Mazutis L, Gilbert J, Ung WL, Weitz DA, Griffiths AD, Heyman JA. Single-cell analysis and sorting using droplet-based microfluidics. Nat Protoc. 2013;8:870-891. 
32. Wang Q, Liu SS, Wang H, Zhu J, Yang Y. Alginate droplets pre-crosslinked in microchannels to prepare monodispersed spherical microgels. Colloid Surface A. 2015;482:371-377.

33. Paques JP, van der Linden E, Sagis LM, van Rijn CJ. Food-grade submicrometer particles from salts prepared using ethanol-in-oil mixtures. J Agric Food Chem. 2012;60:8501-8509.

34. Dang TD, Joo SW. Preparation of tadpole-shaped calcium alginate microparticles with sphericity control. Colloid Surface B. 2013; 102:766-771.
35. Capretto L, Mazzitelli S, Balestra C, et al. Effect of the gelation process on the production of alginate microbeads by microfluidic chip technology. Lab Chip. 2008;8:617-621.

36. Chen XL, Lv HY, Ye M, et al. Novel superparamagnetic iron oxide nanoparticles for tumor embolization application: preparation, characterization and double targeting. Int J Pharm. 2012;426:248-255.

37. Siepmann J, Peppas NA. Modeling of drug release from delivery systems based on hydroxypropyl methylcellulose (HPMC). Adv Drug Deliv Rev. 2012;64:163-174. 


\section{Supplementary materials}
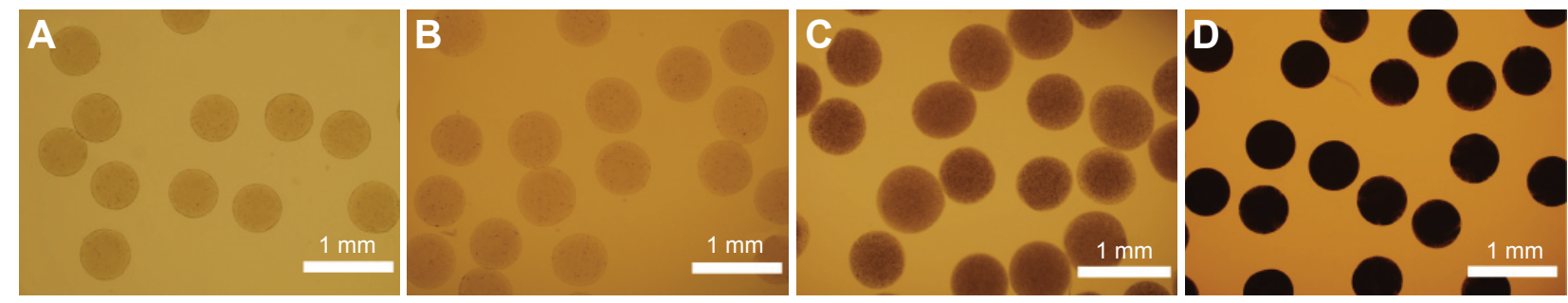

Figure SI Optical photographs of morphology of the SPIO/Ca-ALG microspheres encapsulated with varied contents of SPIO NPs.

Notes: (A) 0; (B) 0.06; (C) 0.6; (D) $6 \mathrm{mg} / \mathrm{mL}$.

Abbreviations: Ca-ALG, calcium alginate; SPIO, superparamagnetic iron oxides; NPs, nanoparticles.

Table SI Characterization of SPIO/Ca-ALG microspheres

\begin{tabular}{llllll}
\hline Content of SPIO $(\mathbf{m g} / \mathbf{m L})$ & $\mathbf{0}$ & $\mathbf{0 . 0 6}$ & $\mathbf{0 . 6 0}$ & $\mathbf{3 . 0}$ & $\mathbf{6 . 0}$ \\
\hline Length/diameter & 1.03 & 1.05 & 1.06 & 1.02 & 1.01 \\
Average diameter $(\mu \mathrm{m})$ & 556 & 618 & 634 & 583 & 546 \\
Coefficient of variation $(\%)$ & 2.7 & 4.6 & 5.3 & 1.9 & 1.9 \\
\hline
\end{tabular}

Abbreviations: SPIO, superparamagnetic iron oxides; Ca-ALG, calcium alginate.

\section{FT-IR spectra}

Figure S2 shows the FT-IR spectra of SPIO NPs, Na-ALG, and SPIO/Ca-ALG microspheres. The peak at $577 \mathrm{~cm}^{-1}$ can be assigned to the $\mathrm{Fe}-\mathrm{O}$ group. The peaks at 1,621 and $1,383 \mathrm{~cm}^{-1}$ are assigned to the $\mathrm{C}=\mathrm{O}$ stretching mode. It is indicative that citrate groups were attached on the surface of SPIO NPs. The wide absorbance near $3,400 \mathrm{~cm}^{-1}$ is assigned to $\mathrm{H}-$ bonded-OH stretching, indicating a large number of hydroxyl groups in Na-ALG and SPIO/Ca-ALG microspheres. The peaks at 1,422 and $1,620 \mathrm{~cm}^{-1}$ in the spectra of Na-ALG can be assigned to the symmetric and asymmetric stretching of carboxyl groups $v_{s}\left(\mathrm{COO}^{-}\right)$, respectively. By contrast, no obvious change was found in the absorption peak of $v_{s}\left(\mathrm{COO}^{-}\right)$of both Na-ALG and SPIO/ $\mathrm{Ca}-\mathrm{ALG}$ microspheres. It is noted that the peak assigned to $v_{\text {as }}\left(\mathrm{COO}^{-}\right)$of Na-ALG shifts from 1,620 to $1,604 \mathrm{~cm}^{-1}$ in the case of SPIO/Ca-ALG microspheres containing $6 \mathrm{mg} / \mathrm{mL}$. In addition, the characteristic peaks of Fe-O in SPIO NPs shift to 595 and $624 \mathrm{~cm}^{-1}$ from $577 \mathrm{~cm}^{-1}$ in case of both SPIO/Ca-ALG microspheres. This is indicative of a strong interaction between citrated modified SPIO NPs and carboxyl and hydroxyl groups in the alginate chain. ${ }^{1}$ This interaction plays an important role to stabilize SPIO NPs in alginate matrix. 


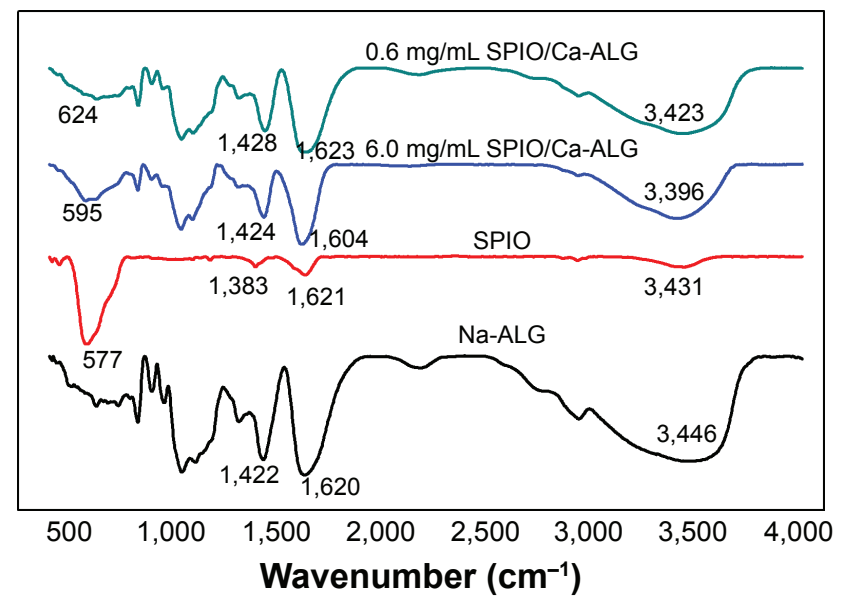

Figure S2 FT-IR spectra of Na-ALG, SPIO NP, and SPIO/Ca-ALG microspheres. Abbreviations: SPIO, superparamagnetic iron oxides; Ca-ALG, calcium alginate; Na-ALG, sodium alginate.

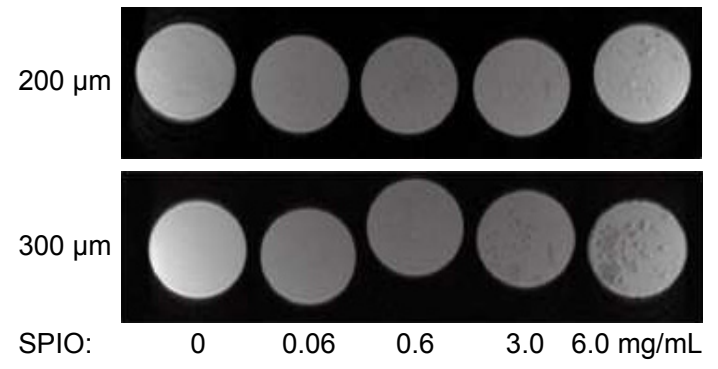

Figure S3 MR images of SPIO/Ca-ALG microspheres with a size of 200 and $300 \mu \mathrm{m}$ containing various contents of SPIO NPs.

Abbreviations: MR, magnetic resonance; SPIO, superparamagnetic iron oxides; NPs, nanoparticles; Ca-ALG, calcium alginate.

\section{Chemical structure of doxorubicin hydrochloride, 5-fluorouracil and alginate}

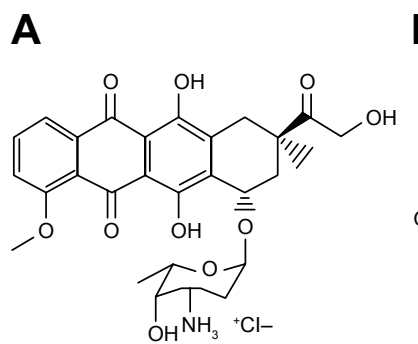

B C

Figure S4 Chemical structures of (A) Doxorubicin hydrochloride; (B) 5-fluorouracil; (C) alginate composed with the blocks of I,4-linked $\alpha$-D-mannuronate (M) and $\beta$-Lguluronate $(G)$.

\section{Reference}

1. Ma HL, Qi XR, Maitani Y. Preparation and characterization of superparamagnetic iron oxide nanoparticles stabilized by alginate. Int J Pharm. 2007;333:177-186. 
International Journal of Nanomedicine

Dovepress

\section{Publish your work in this journal}

The International Journal of Nanomedicine is an international, peerreviewed journal focusing on the application of nanotechnology in diagnostics, therapeutics, and drug delivery systems throughout the biomedical field. This journal is indexed on PubMed Central, MedLine, CAS, SciSearch ${ }^{\circledR}$, Current Contents ${ }^{\circledR} /$ Clinical Medicine,
Journal Citation Reports/Science Edition, EMBase, Scopus and the Elsevier Bibliographic databases. The manuscript management system is completely online and includes a very quick and fair peer-review system, which is all easy to use. Visit http://www.dovepress.com/ testimonials.php to read real quotes from published authors.

Submit your manuscript here: http://www.dovepress.com/international-journal-of-nanomedicine-journal 